\title{
TD/GC-MS analysis of volatile markers emitted from mono- and co- cultures of Enterobacter cloacae and Pseudomonas aeruginosa in artificial sputum
}

\author{
Oluwasola Lawal ${ }^{1,4}$ (D) Hugo Knobel ${ }^{3} \cdot$ Hans Weda $^{2} \cdot$ Tamara M. E. Nijsen $^{2} \cdot$ Royston Goodacre $^{4}(\mathbb{D} \cdot$ \\ Stephen J. Fowler ${ }^{1,5}$ on behalf of the BreathDx consortium
}

Received: 1 February 2018 / Accepted: 26 March 2018 / Published online: 26 April 2018

(c) The Author(s) 2018

\begin{abstract}
Introduction Infections such as ventilator-associated pneumonia (VAP) can be caused by one or more pathogens. Current methods for identifying these pathogenic microbes often require invasive sampling, and can be time consuming, due to the requirement for prolonged cultural enrichment along with selective and differential plating steps. This results in delays in diagnosis which in such critically ill patients can have potentially life-threatening consequences. Therefore, a non-invasive and timely diagnostic method is required. Detection of microbial volatile organic compounds (VOCs) in exhaled breath is proposed as an alternative method for identifying these pathogens and may distinguish between mono- and poly-microbial infections.

Objectives To investigate volatile metabolites that discriminate between bacterial mono- and co-cultures.

Methods VAP-associated pathogens Enterobacter cloacae and Pseudomonas aeruginosa were cultured individually and together in artificial sputum medium for $24 \mathrm{~h}$ and their headspace was analysed for potential discriminatory VOCs by thermal desorption gas chromatography-mass spectrometry.

Results Of the 70 VOCs putatively identified, 23 were found to significantly increase during bacterial culture (i.e. likely to be released during metabolism) and 13 decreased (i.e. likely consumed during metabolism). The other VOCs showed no transformation (similar concentrations observed as in the medium). Bacteria-specific VOCs including 2-methyl-1-propanol, 2-phenylethanol, and 3-methyl-1-butanol were observed in the headspace of axenic cultures of E. cloacae, and methyl 2-ethylhexanoate in the headspace of $P$. aeruginosa cultures which is novel to this investigation. Previously reported VOCs 1-undecene and pyrrole were also detected. The metabolites 2-methylbutyl acetate and methyl 2-methylbutyrate, which are reported to exhibit antimicrobial activity, were elevated in co-culture only.

Conclusion The observed VOCs were able to differentiate axenic and co-cultures. Validation of these markers in exhaled breath specimens could prove useful for timely pathogen identification and infection type diagnosis.
\end{abstract}

Keywords Bacteria $\cdot$ Enterobacter cloacae $\cdot$ Gas Chromatography-Mass Spectrometry $\cdot$ Infection $\cdot$ Pseudomonas aeruginosa $\cdot$ Volatile organic compounds

Electronic supplementary material The online version of this article (https://doi.org/10.1007/s11306-018-1357-5) contains supplementary material, which is available to authorized users.

Stephen J. Fowler

stephen.fowler@manchester.ac.uk

1 Division of Infection, Immunity and Respiratory Medicine, School of Biological Sciences, Faculty of Biology, Medicine and Health, The University of Manchester, Manchester, UK

2 Philips Research, Royal Philips B.V., Eindhoven, The Netherlands
3 Philips Innovation Labs, Philips Lighting, Eindhoven, The Netherlands

4 School of Chemistry, Manchester Institute of Biotechnology, University of Manchester, Manchester, UK

5 Manchester Academic Health Science Centre, The University of Manchester and Manchester University NHS Foundation Trust, Manchester, UK 


\section{Introduction}

Lower respiratory tract infections such as pneumonia occur frequently in patients in intensive care units (ICUs), and can be mono- or poly-microbial (Kalanuria et al. 2014; Ferrer et al. 2015). Patients are generally first administered empirical antibiotics while identity of the causal pathogen(s) is confirmed. Full microbial identification can take up to 7 days with the potential for interim inappropriate antibiotic regimens, and this lack of appropriate antibiotic therapy guidance can lead to poor clinical outcomes (due to lack of efficacy), increased length of stay, and unnecessary antibiotic resistance (Combes et al. 2002; Ferrer et al. 2015; Mietto et al. 2013). In addition to being time consuming, the procedure for obtaining biological specimens from patients is often invasive. Thus, early and accurate detection of infection is a critical ambition for optimised patient management and antimicrobial stewardship on the ICU.

The analysis of VOCs in exhaled breath is proposed as a potential alternative diagnostic tool (Phillips 1992; Lourenco and Turner 2014; Ahmed et al. 2017; Lawal et al. 2017a). It is postulated that VOCs from causal pathogens of infection are exhaled and thus provides potential for early non-invasive detection. Bacterial VOCs may be investigated by means of headspace analysis of in vitro cultures. These VOCs are commonly 'trapped' on thermal desorption tubes and analysed by gas chromatography-mass spectrometry (GC-MS) (Boots et al. 2014; Neerincx et al. 2016; Filipiak et al. 2012). Other pre-concentration methods such as solid phase microextraction (Tait et al. 2014; Shestivska et al. 2011) and needle trap devices (Zscheppank et al. 2014) have been described. Online analysis which does not require prior sample enrichment has also been reported (Allardyce et al. 2006).

Whilst there has been a wealth of information regarding VOCs from bacterial mono-cultures, there is limited information regarding volatile metabolites from microbial co-cultures. Neerincx et al. have reported VOCs from $P$. aeruginosa and Aspergillus fumigatus co-culture (2016) and Zhu et al. cultured the former pathogen with Staphylococcus aureus (Zhu et al. 2010). Microorganisms associated with poly-microbial infections have been reported in literature (Ferrer et al. 2015). A common combination includes a clinically relevant member of the Enterobacteriaceae family such as Enterobacter cloacae, and a non-fermenting Gram-negative rod-shaped bacterium for example Pseudomonas aeruginosa (Davin-Regli and Pages 2015; John et al. 1982). E. cloacae is commonly found in the lower gastrointestinal tract and antibiotic administration has been linked to its overgrowth resulting in translocation and pathogenicity (Park 2005). P. aeruginosa is ubiquitously present in soil and water and is an opportunistic pathogen which thrives when host defense mechanisms are impaired. To investigate potential discriminatory mono- and co-culture VOC markers, E. cloacae and $P$. aeruginosa were cultured separately and together in artificial sputum medium (ASM), in order to mimic the clinical diagnostic specimen. In addition, we have previously shown the influence of medium substrates on VOC profiles (Lawal et al. 2017b). The use of ASM culminated in the detection of diverse VOCs justifying its use in this study. VOCs in the headspace of bacterial cultures were trapped onto sorbent tubes and analysed by thermal desorption gas chromatography (TD-GC/MS). In addition, we seek to explore the growth relationship of both microbes. By performing this in vitro investigation, observed changes in VOC profile may aid in distinguishing between types of infections and may translate towards improved antibiotic therapy guidance.

\section{Materials and methods}

\subsection{Media preparation}

Tryptic soy agar (TSA), was made by combining tryptic soy broth (Oxoid, Basingstone, UK) and Agar bacteriological (Agar NO. 1, Oxoid) as specified by the manufacturer. Levine EMB agar (LEA) (Fluka Analytical, UK) was prepared according to manufacturer's specification, and details for ASM preparation can be found in (Diraviam Dinesh 2010). Briefly to prepare $1 \mathrm{~L}$ of ASM, type II mucin (5 g; Sigma-Aldrich, Germany), salmon sperm DNA (4 g; Sigma-Aldrich, Germany), diethylenetriaminepentaacetic acid (5.9 mg; Sigma-Aldrich, Germany), sodium chloride ( $5 \mathrm{~g}$; Sigma-Aldrich, Germany), potassium chloride ( $2.2 \mathrm{~g}$; Sigma-Aldrich, Germany), Tris base (1.8 g; Sigma-Aldrich, Germany), egg yolk emulsion (5 mL; Oxoid), casamino acids ( $5 \mathrm{~g}$; BD, Sparks, USA) were all dissolved in distilled water and subsequently autoclaved at $121^{\circ} \mathrm{C}$ for $15 \mathrm{~min}$.

\subsection{Bacterial culture}

E. cloacae, DSM 30054 and P. aeruginosa, ATCC 10145 were used in this study. The strains were retrieved from glycerol frozen stock, sub-cultured twice on TSA plates to ensure purity and incubated overnight at $37{ }^{\circ} \mathrm{C}$ to obtain axenic colonies. Single colonies were subsequently transferred into $50 \mathrm{~mL}$ ASM in $250 \mathrm{~mL}$ Schott Duran glass bottles and incubated in an orbital shaker (innova ${ }^{\circledR} 40$ incubator shaker series, New Brunswick Scientific) at $37^{\circ} \mathrm{C}$ with $200 \mathrm{rpm}$ shaking to obtain overnight cultures. To investigate the growth relationship of the microorganisms and determine a time point for headspace sampling, approximately $10^{6}$ cells 
of each bacterium were added into the same glass bottle containing $50 \mathrm{~mL}$ ASM and incubated at $37{ }^{\circ} \mathrm{C}$ in an orbital shaker (innova ${ }^{\circledR} 40$ ) for $24 \mathrm{~h}$. Corresponding mono-cultures were also established separately with the same amount of cells. To establish viable cell numbers, $1 \mathrm{~mL}$ aliquots were collected at 5, 8, 11, and $24 \mathrm{~h}$ from the distinct cultures and mixed with $9 \mathrm{~mL}$ physiological saline $(0.9 \% \mathrm{NaCl})$. A classical microbiological plating approach was then performed which involved an initial dilution series and spreading of diluted homogeneous sample $(100 \mu \mathrm{L})$ on LEA. The plates were then incubated at $37^{\circ} \mathrm{C}$ for $24 \mathrm{~h}$ and the viable bacteria were noted as colony forming units (Stuart ${ }^{\circledR}$ colony counter, Barloworld Scientific Limited Stone, Staffordshire, UK). LEA is a selective and differential medium which allows the growth of Gram-negative bacterium and also produces different phenotypes for distinct microbes based on substrate conversions. In addition, $1 \mathrm{~mL}$ of bacterial cultures was collected for corresponding optical density $\left(\mathrm{OD}_{600}\right)$ measurements (Eppendorf BioPhotometer plus, Eppendorf AG, Hamburg, Germany).

\subsection{Bacterial culture headspace sampling}

Liquid mono- and co-cultures were prepared as described above. After incubation, and still positioned in the incubator to minimise condensation effects, the bottles containing the bacterial cultures were purged with dry nitrogen at a flow rate of $60 \mathrm{~mL} / \mathrm{min}$ through customised caps (GL45 caps, Fischer scientific, UK). The headspace was simultaneously evacuated through a different outlet and again mixed with dry nitrogen introduced at a flow rate of $140 \mathrm{~mL} / \mathrm{min}$ to lower the relative humidity below $100 \%$ at room temperature, thus minimising condensation, and was subsequently trapped onto custom made thermal desorption multi-bed sorbent tubes containing Tenax GR-Carbograph 5TD sorbents (Markes, Llantrisant, UK) using a pump at a flow rate of $200 \mathrm{~mL} / \mathrm{min}$ for $6 \mathrm{~min}$. Water vapour is known to interfere with the quantitative capture of VOCs hence the need to reduce its effects using dry nitrogen. The headspace was collected at $24 \mathrm{~h}$ as adequate growth was observed for both bacterium in co-culture. The sorbent tubes were purged with dry nitrogen again after sampling at a flow rate of $100 \mathrm{~mL} /$ $\min$ for $6 \mathrm{~min}$.

\subsection{Gas chromatography-mass spectrometry (GC- MS) analysis}

Internal calibration standards were spiked onto the sorbent tubes by dilution of gaseous calibration standards (10 ppmv acetone- $d_{6}$, hexane- $d_{14}$, toluene- $d_{8}$, xylene- $d_{10}$ in nitrogen, Air Products, Amsterdam, The Netherlands) using a custommade dilution system. As a quality assurance procedure, empty and sorbent-only tubes are also analysed. Sorbent tubes were thermally desorbed at $225^{\circ} \mathrm{C}$ (TDSA, Gerstel, Mülheim an der Ruhr, Germany) into the packed liner. Solvent venting mode was used to transfer the sample to the packed liner (filled with Tenax TA) held at $-55^{\circ} \mathrm{C}$ which was subsequently heated to $280{ }^{\circ} \mathrm{C}$ to transfer the VOCs into the GC capillary column. A cold trap (CTS2, Gerstel, Mülheim an der Ruhr, Germany) was used to minimise band broadening (initial temperature $-150{ }^{\circ} \mathrm{C}$, after $1.6 \mathrm{~min}$ heated to $\left.220^{\circ} \mathrm{C}\right)$. A capillary gas chromatograph $(7890 \mathrm{~N}$ GC, Agilent, Santa Clara, CA, USA) using a VF1-MS column $(30 \mathrm{~m} \times 0.25 \mathrm{~mm}$, film thickness $1 \mu \mathrm{m}, 100 \%$ dimethylpolysiloxane, Varian Chrompack, Middelburg, The Netherlands) was used with the following temperature program: $30{ }^{\circ} \mathrm{C}$, hold $3.5 \mathrm{~min}$, ramp $5{ }^{\circ} \mathrm{C} / \mathrm{min}$ to $50{ }^{\circ} \mathrm{C}$, hold $0 \mathrm{~min}$, ramp $10{ }^{\circ} \mathrm{C} / \mathrm{min}$ to $90^{\circ} \mathrm{C}$, hold $0 \mathrm{~min}$, ramp $15^{\circ} \mathrm{C} / \mathrm{min}$ to $130{ }^{\circ} \mathrm{C}$, hold $0 \mathrm{~min}$, ramp $30^{\circ} \mathrm{C} / \mathrm{min}$ to $180^{\circ} \mathrm{C}$, hold $0 \mathrm{~min}$, ramp $40{ }^{\circ} \mathrm{C} / \mathrm{min}$ to $280{ }^{\circ} \mathrm{C}$, hold $1 \mathrm{~min}$. A Time of Flight mass spectrometer (LECO Pegasus 4D system, LECO, St. Joseph, MI, USA) was used in electron ionization mode at $70 \mathrm{eV}$, with a scan range of $m / z 29-400 \mathrm{Da}$, scanning rate 20 scans/s.

\subsection{Data processing and analysis}

\subsubsection{Data pre-processing}

LECO Chromatof software (LECO, St. Joseph, MI, USA) was used to acquire, analyse, and manage GC-MS runs. The acquired chromatograms can be viewed using this software and mass spectra from individual peaks cross-referenced with National Institute of Standards and Technology library 14 (NIST, Gaithersburg, MD, USA) for putative identification purposes, and followed the metabolomics standards initiative (MSI) guidelines for metabolite identification (Sumner et al. 2007). Metabolites with a NIST match factor of $\geq 800$ were investigated.

Raw data in manufacturer's data format were converted into a netCDF format utilising the LECO Chromatof software. All statistical analyses were performed using the $\mathrm{R}$ software (version 3.4.2; R Core Team (2017)). The $x \mathrm{cms}$ package following the approach as outlined by Smith et al. (2006) was used to pre-process the netCDF files in R. The product of raw data pre-processing is a data file containing ion fragments, their corresponding $\mathrm{m} / \mathrm{z}$, retention times, and also integrated areas. Normalisation using the internal standard (IS), toluene- $d_{8}$, was based on the $100 \mathrm{~m} / z$ parent ion.

\subsection{Univariate and multivariate analyses}

For univariate analysis when applicable, the non-parametric Kruskal-Wallis test was performed. Games-Howell post-hoc test was then subsequently used to investigate statistically significant entities. A critical $\alpha=0.05$ value was used in 
tests. False discovery rate method was used to adjust the obtained $p$-values after multiple hypothesis testing.

Principal component-discriminant function analysis (PC-DFA) was used for multivariate exploration of data (Jombart et al. 2010; Goodacre et al. 1998). Briefly, the aim is to maximise the variance between groups and minimise variance within groups. Principal components (PCs) are the input variables to DFA therefore PCA on the X data (VOC profiles) precedes DFA; the R package adegenet was used for this analysis (Jombart 2008). For validation, the dataset was divided into $70 \%$ training and $30 \%$ test sets. A PC-DFA model was built using the training set and the test set was then projected into the subspace created by the training set to visualise the prediction of the test data on the basis of proximity to the training samples originating from the same bacterial groups.

\section{Results}

\subsection{Appearance of E. cloacae and P. aeruginosa on Levine EMB agar}

Levine EMB agar was used as a selective and differential medium to distinguish between the bacteria species under investigation. E. cloacae appeared to develop dark pink colonies and colourless/white colonies were observed for $P$. aeruginosa (Fig. 1).

\subsection{E. cloacae and P. aeruginosa growth curves in mono-versus co-cultures}

E. cloacae and $P$. aeruginosa were grown individually and together. To determine viable cell numbers, a serial dilution of individual cultures was performed and then plated on Levine EMB agar. E. cloacae mono- and co-cultures were observed to have a similar growth pattern and enter the log phase quicker than $P$. aeruginosa which displayed

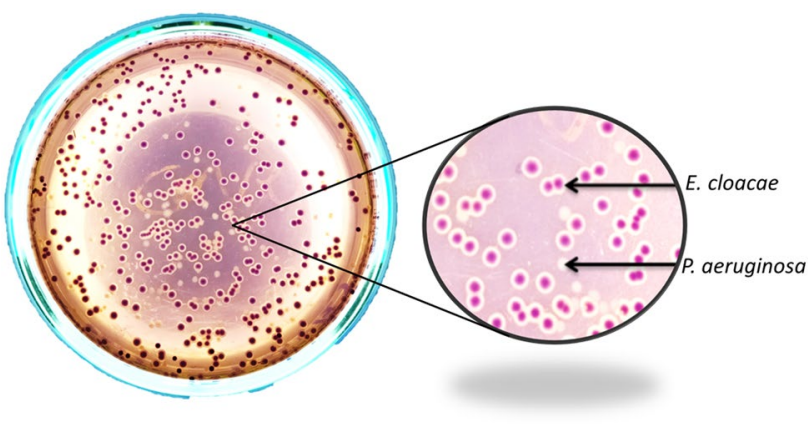

Fig. 1 Phenotypes of E. cloacae (dark pink) and P. aeruginosa (white/colourless) colonies on selective and differential medium Levine EMB agar a prolonged lag period especially in the co-culture before entering the growth phase (Fig. 2a). Optical densities were also measured to complement the viable cell counts (Fig. 2b).

\subsection{VOC profiling in bacterial cultures}

VOCs originating specifically from bacterial cultures (absent in medium control) are shown in Fig. 3. Metabolites that were found to be present in both the headspace of bacterial cultures and blank medium but at levels that are significantly different between the two after performing post-hoc analyses with correction by false discovery rate are shown in Table 1. Hypothesis testing was performed to compare the concentrations of the VOCs in the headspace of bacterial cultures and medium control and differences indicate whether the VOC was released or consumed. Other identified VOCs are shown in Table 2. VOCs that were observed to be elevated in the bacterial co-culture headspace are shown in Fig. 4. These are putatively identified compounds based on fragmentation pattern comparison to the NIST reference library and are therefore MSI level 2 (Sumner et al. 2007). Representative chromatograms for axenic cultures and co-cultures are shown in the supplementary material (Fig S1).

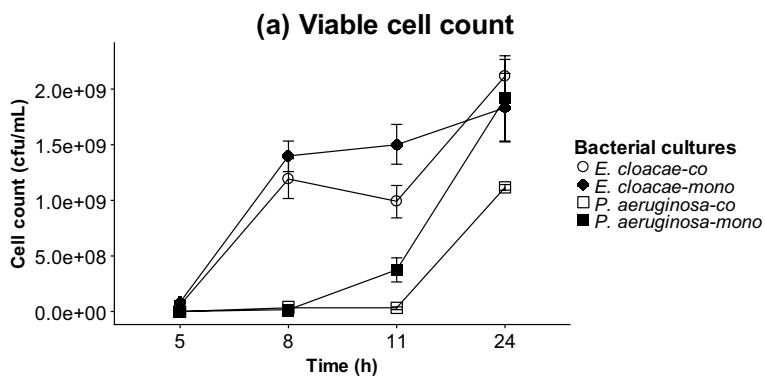

(b) Optical density (OD)

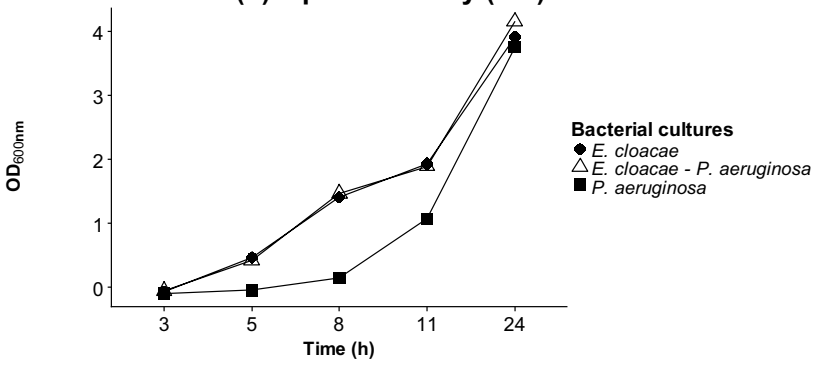

Fig. 2 a Viable cell counts expressed as colony forming units per $\mathrm{mL}$ (cfu/mL), b optical densities of mono- and co-cultures of $E$. cloacae and $P$. aeruginosa over a period of $24 \mathrm{~h}$. Viable cell counts were performed in triplicates and the error bars represent standard deviations. Levine EMB agar and artificial sputum were used as solid and liquid media respectively 
Fig. 3 Boxplots showing the internal standard (IS) normalised concentrations of bacteria-specific VOCs in the medium blank, E. cloacae (Ec), P. aeruginosa $(\mathrm{Pa})$, and $P$. aeruginosa-E. cloacae $(\mathrm{Pa}-\mathrm{Ec})$ bacterial cultures. The boxplots were generated from eight repeats

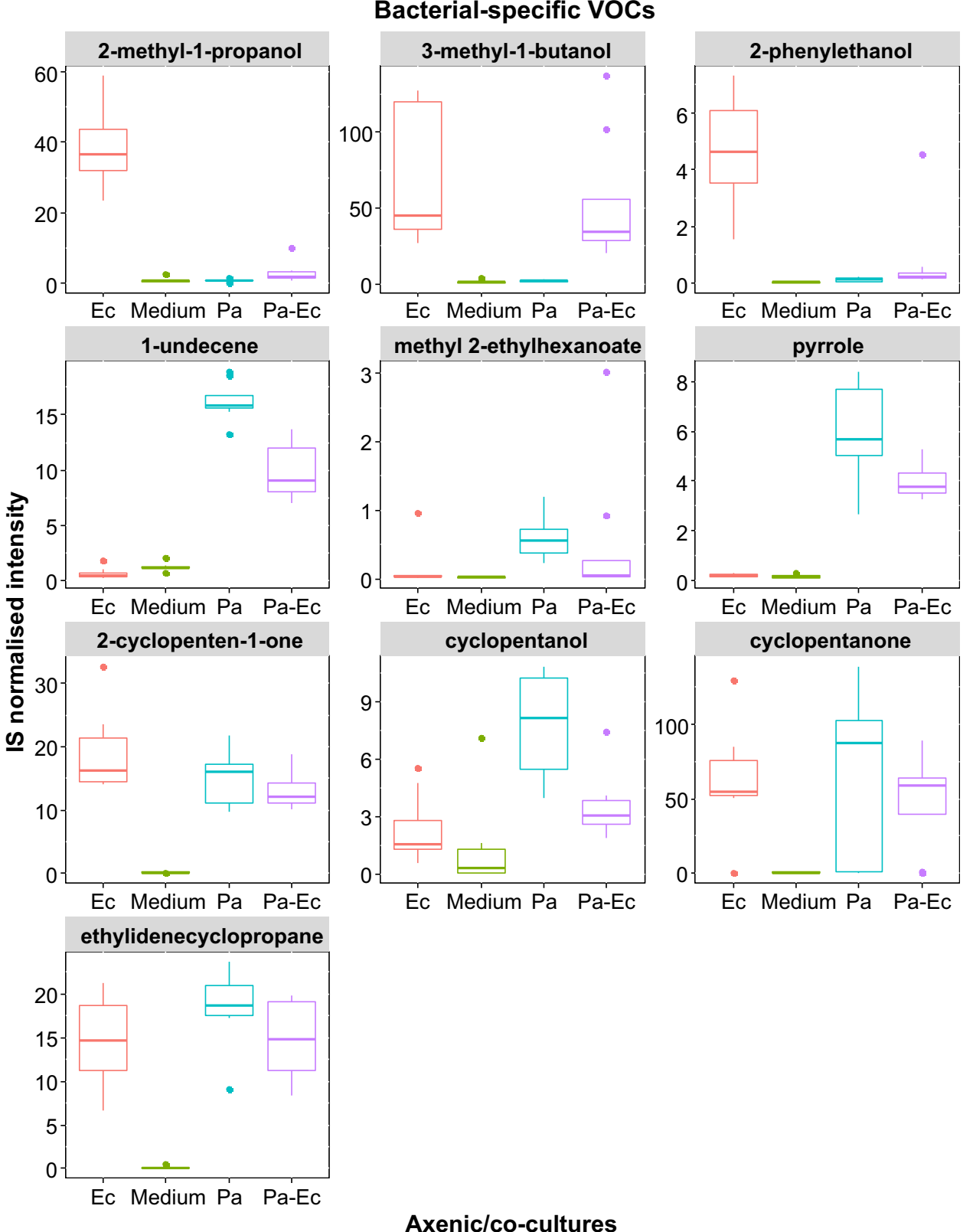

\subsection{Multivariate analysis}

PC-DFA was implemented to visualise the data in dimensional space and discern VOCs that discriminate between respective mono- and co-cultures. Eight PCs were included for DFA as it achieved the lowest root mean square error after performing cross validation. This proportion accounted for approximately $64.5 \%$ of conserved variance extracted from the dataset (Fig S2). The training set was used to build the model and the test set to evaluate its validity through projection into subspace established by the training set. The test data were congruent with their respective training set clusters (Fig. 5). From the loadings plot (Fig S3), fragments from 2-methyl-1-propanol and 1-undecene were observed to contribute to the separation between $E$. cloacae monoculture and $P$. aeruginosa mono- and co-cultures along DF1. Along DF2, the co-culture appears to be separated from both mono-cultures with fragments emanating from 3-methyl1-butanol, isoamyl butyrate, and 2-methylbutyl acetate.

\section{Discussion}

The viable cell count indicated maximal growth of both microbes after $11 \mathrm{~h}$. Several VOCs were observed in the headspace of axenic cultures and co-cultures. Some 
Table 1 VOCs detected in bacterial cultures and blank medium control is shown

\begin{tabular}{lllllll}
\hline VOC & CAS\# & $m / z$ & Ec & Pa & Pa-Ec & Class \\
\hline 2-Ethyl-trans-2-butenal & $63883-69-2$ & $98\left(\mathrm{M}^{+}\right)$ & $\downarrow$ & $\downarrow$ & $\downarrow$ & Aldehyde \\
Benzaldehyde & $100-52-7$ & $77(\mathrm{bp})$ & $\downarrow$ & $\downarrow$ & $\downarrow$ & \\
2-Methylbutanal & $96-17-3$ & $41(\mathrm{bp})$ & $\mathrm{ns}$ & $\downarrow$ & $\downarrow$ & \\
Furfural & $98-01-1$ & $96(\mathrm{bp})\left(\mathrm{M}^{+}\right)$ & $\mathrm{ns}$ & $\downarrow$ & $\downarrow$ & \\
Hexanal & $66-25-1$ & $44(\mathrm{bp})$ & $\downarrow$ & $\downarrow$ & $\downarrow$ & \\
2-Ethylhexenal & $645-62-5$ & $126\left(\mathrm{M}^{+}\right)$ & $\downarrow$ & $\downarrow$ & $\downarrow$ & \\
2-Methylpropanal & $78-84-2$ & $43(\mathrm{bp})$ & $\downarrow$ & $\downarrow$ & $\downarrow$ & \\
2,2-Dimethylpropanal & $630-19-3$ & $86\left(\mathrm{M}^{+}\right)$ & $\downarrow$ & $\downarrow$ & $\mathrm{ns}$ & \\
1-Butanol & $71-36-3$ & $56(\mathrm{bp})$ & $\downarrow$ & $\downarrow$ & $\mathrm{ns}$ & Alcohol \\
2-Butanol & $78-92-2$ & $45(\mathrm{bp})$ & $\mathrm{ns}$ & $\uparrow$ & $\mathrm{ns}$ & \\
2-Furanmethanol & $98-00-0$ & $98(\mathrm{bp})$ & $\downarrow$ & $\downarrow$ & $\downarrow$ & \\
2-Propanol & $67-63-0$ & $43(\mathrm{fg})$ & $\mathrm{ns}$ & $\uparrow$ & $\mathrm{ns}$ & \\
Ethanol & $64-17-5$ & $31(\mathrm{bp})$ & $\downarrow$ & $\downarrow$ & $\downarrow$ & \\
Pentane & $109-66-0$ & $43(\mathrm{bp})$ & $\downarrow$ & $\downarrow$ & $\downarrow$ & Alkane \\
Ethyl propionate & $105-37-3$ & $102\left(\mathrm{M}^{+}\right)$ & $\uparrow$ & $\downarrow$ & $\uparrow$ & Ester \\
2-Methylfuran & $534-22-5$ & $82(\mathrm{bp})$ & $\uparrow$ & $\mathrm{ns}$ & $\uparrow$ & Heterocyclic \\
Acetone & $67-64-1$ & $43(\mathrm{bp})$ & $\uparrow$ & $\uparrow$ & $\uparrow$ & Ketone \\
2-Heptanone & $110-43-0$ & $58(\mathrm{fg})$ & $\uparrow$ & $\mathrm{ns}$ & $\mathrm{ns}$ & \\
2,6-Dimethyl pyrazine & $108-50-9$ & $108(\mathrm{bp})\left(\mathrm{M}^{+}\right)$ & $\uparrow$ & $\mathrm{ns}$ & $\uparrow$ & Nitrogen-containing compound \\
Dimethyl sulfide & $75-18-3$ & $62(\mathrm{bp})$ & $\uparrow$ & $\mathrm{ns}$ & $\mathrm{ns}$ & Sulfur-containing compound \\
Dimethyl disulfide & $624-92-0$ & $94(\mathrm{bp})$ & $\uparrow$ & $\uparrow$ & $\uparrow$ & \\
Methylthioacetate & $1534-08-3$ & $90(\mathrm{fg})$ & $\uparrow$ & $\mathrm{ns}$ & $\uparrow$ & \\
\hline
\end{tabular}

$\left(\mathrm{M}^{+}\right)$molecular ion, $b p$ base peak, $f g$ fragment

Unless depicted with ns (non-significant), $(\uparrow)$ and $(\downarrow)$ assigned to bacterial cultures indicate a statistically significant (after false discovery rate correction) increase and decrease respectively in headspace concentration in comparison to medium control. The normalised abundances of representative fragments $(\mathrm{m} / \mathrm{z})$ were used for hypothesis testing metabolites (i.e. 3-methyl-1-butanol, 1-undecene) from axenic cultures were also present in the co-culture headspace while others appear diminished (2-methyl-1-propanol, 2-phenylethanol). Furthermore, some VOCs with very low levels in the mono-cultures were upregulated in the co-culture headspace (methyl 2-methylbutyrate, isoamyl butyrate, and 2-methylbutyl acetate).

The growth dynamic in both mono- and co-cultures of the bacteria was explored by a serial dilution plating method. Levine EMB agar, a selective (allows growth of Gram-negative and suppresses the growth of Gram-positive bacteria) and differential (distinct appearance of colonies based on metabolism of different nutrients) medium was used to discriminate bacterial colonies (Leininger et al. 2001; Parisi and Marsik 1969; Gehm and Heukelekian 1935). In this case E. cloacae is capable of metabolising lactose into lactic acid which reduces the $\mathrm{pH}$ of the medium thus resulting in the appearance of dark pink colonies (Leininger et al. 2001). In contrast, $P$. aeruginosa is incapable of conducting the aforementioned conversion and instead metabolises peptone which increases the $\mathrm{pH}$ of the medium and appear as colourless/white colonies. The observed distinct phenotypes aided in distinguishing the bacterial species for growth monitoring.

The reporting of volatile metabolites originating from E. cloacae is scarce in literature. VOCs such as 2-methyl1-propanol, 3-methyl-1-butanol, and 2-phenylethanol were observed to be specific for this microbe in this study. These metabolites have been reported to be released by other VAPassociated bacterial species (Tait et al. 2014; Filipiak et al. 2012; Junger et al. 2012) thus the use of a sole marker may not be suitable and may require a broad range of selected metabolites to aid microbial identification. VOCs emitted by $P$. aeruginosa have been previously communicated which include 1-undecene and pyrrole which were also observed in this investigation (Filipiak et al. 2012). Methyl 2-ethylhexanoate has not previously been reported. Bacteria specific VOCs such as cyclopentanone and cyclopentanol emitted by both microbes were also observed and have been reported (Lawal et al. 2017b).

In addition to the metabolites measured only in bacterial cultures, some VOCs were observed in both blank medium and bacterial cultures and may be useful. The statistically significant compounds can be separated from the redundant 
Table 2 Other (statistically non-significant) VOCs detected in the headspace of bacterial cultures with respect to blank medium control

\begin{tabular}{|c|c|c|c|}
\hline VOC & CAS\# & $m / z$ & Class \\
\hline 2-Butenal & $4170-30-3$ & $41(b p)$ & Aldehyde \\
\hline Acetaldehyde & $75-07-0$ & 29 (bp) & \\
\hline Methacrolein & $78-85-3$ & $41(\mathrm{bp})$ & \\
\hline Pentanal & $110-62-3$ & 44 (bp) & \\
\hline 2-Ethyl-1-hexanol & $104-76-7$ & $55(\mathrm{fg})$ & Alcohol \\
\hline Dodecane & $112-40-3$ & $57(\mathrm{bp})$ & Alkane \\
\hline 2,4-Dimethylheptane & $2213-23-2$ & $43(\mathrm{bp})$ & \\
\hline 2,3,5-Trimethylhexane & $1069-53-0$ & 43 (bp) & \\
\hline Nonane & $111-84-2$ & 43 (bp) & \\
\hline 4-Methylheptane & $589-53-7$ & $43(\mathrm{bp})$ & \\
\hline 2,3-Dimethylbutane & $79-29-8$ & $43(\mathrm{bp})$ & \\
\hline Decane & $124-18-5$ & $57(b p)$ & \\
\hline Heptane & $142-82-5$ & 43 (bp) & \\
\hline Octane & $111-65-9$ & 43 (bp) & \\
\hline 2-Methylpentane & $107-83-5$ & 43 (bp) & \\
\hline 2,4-Dimethylhexane & $589-43-5$ & 43 (bp) & \\
\hline 1,3-Dimethylbenzene & $108-38-3$ & 91 (bp) & Aromatic hydrocarbon \\
\hline 1,2,3-Trimethylbenzene & $526-73-8$ & 105 (bp) & \\
\hline Ethylbenzene & $100-41-4$ & $91(b p)$ & \\
\hline m-Di-tert-butylbenzene & $1014-60-4$ & 175 (bp) & \\
\hline Benzene & $71-43-2$ & $78(\mathrm{bp})\left(\mathrm{M}^{+}\right)$ & \\
\hline$\alpha$-Pinene & $80-56-8$ & $93(\mathrm{bp})$ & Alkene \\
\hline 2-Butene & $107-01-7$ & $41(\mathrm{bp})$ & \\
\hline 2-Methyl-1-pentene & $763-29-1$ & $56(\mathrm{bp})$ & \\
\hline 2,4-Dimethyl-1-heptene & $19549-87-2$ & 43 (bp) & \\
\hline Ethyl tert-butyl ether & $637-92-3$ & $59(\mathrm{bp})$ & Ether \\
\hline 2-Ethylfuran & $3208-16-0$ & $81(b p)$ & Heterocyclic \\
\hline 2,4-Dimethylfuran & $3710-43-8$ & $96(\mathrm{bp})\left(\mathrm{M}^{+}\right)$ & \\
\hline 2-Pentylfuran & $3777-69-3$ & $81(\mathrm{bp})$ & \\
\hline Furan & $110-00-9$ & $68(\mathrm{bp})\left(\mathrm{M}^{+}\right)$ & \\
\hline 2-Butanone & $78-93-3$ & $43(\mathrm{bp})$ & Ketone \\
\hline 2-Pentanone & $107-87-9$ & $43(\mathrm{bp})$ & \\
\hline 3-Hexen-2-one & $763-93-9$ & $83(\mathrm{bp})$ & \\
\hline 2-Methylthiophene & $554-14-3$ & 97 (bp) & Sulfur-containing compound \\
\hline Carbon disulfide & $75-15-0$ & $76(\mathrm{bp})$ & \\
\hline Dimethyl trisulfide & $3658-80-8$ & $126(\mathrm{bp})\left(\mathrm{M}^{+}\right)$ & \\
\hline Thiophene & $110-02-1$ & $84(\mathrm{bp})\left(\mathrm{M}^{+}\right)$ & \\
\hline
\end{tabular}

The normalised concentrations of representative fragments $(\mathrm{m} / \mathrm{z})$ were used for hypothesis testing $\left(M^{+}\right)$molecular ion, $b p$ base peak, $f g$ fragment components and may potentially prove to be a reliable addition to bacteria-specific VOCs when compiling a panel of biomarkers for microbial identification. Other sterile media VOCs especially aldehydes were observed to be consumed. This consumption has previously being reported for $P$. aeruginosa (Filipiak et al. 2012). This phenomenon was also observed for alcohols except for 2-butanol which appears to be released by $P$. aeruginosa.

Also novel to our study was the investigation of potential markers for co-habitation of two pathogenic microbes.
Commensal microbes are ubiquitously distributed in humans and have an important role in limiting the influence of 'foreign' potentially pathogenic microflora (Martin et al. 2013; Littman and Pamer 2011; Ubeda et al. 2017). E. cloacae is found in the environment and also known to be a member of the gut flora of a healthy host and is considered a commensal microbe (Keller et al. 1998; Davin-Regli and Pages 2015). However, it is implicated as an opportunistic pathogen in VAP and evidence suggests that the endogenous reservoir of this microbe is the 
Fig. 4 Boxplots showing the internal standard (IS) normalised concentrations of methyl 2-methylbutyrate, isoamyl butyrate, and 2-methylbutyl acetate in headspace of blank medium, E. cloacae (Ec), $P$. aeruginosa $(\mathrm{Pa})$, and $P$. aeruginosa-E. cloacae $(\mathrm{Pa}-\mathrm{Ec})$ bacterial cultures. These VOCs were found to be elevated in the co-culture samples in comparison to axenic cultures and blank medium. After performing Kruskal-Wallis test, GamesHowell test was used for posthoc analyses and $p$-values were corrected by false discovery rate. The boxplots were generated from eight repeats

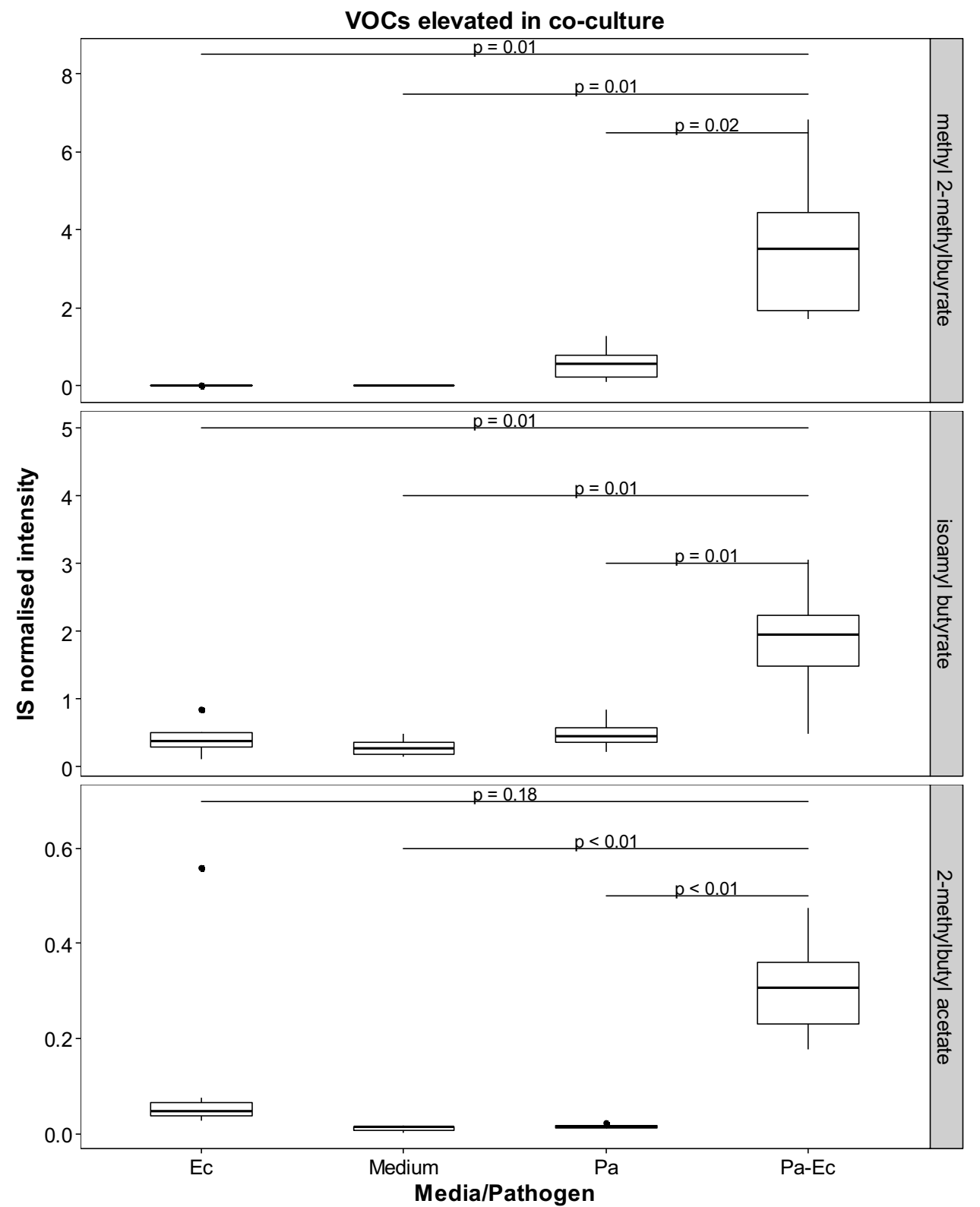

predominant source as a result of translocation from the gut to the lung (Keller et al. 1998; Park 2005). Therefore in conjunction with opportunistic pathogens such as $P$. aeruginosa it may culminate in a poly-microbial infection in a host. E. cloacae appears to enter the growth phase in co-culture in a shorter period (after $5 \mathrm{~h}$ ) in comparison to $P$. aeruginosa which needed approximately $11 \mathrm{~h}$ before entering the log phase. This prolonged lag phase may be as a result of a larger genome size in comparison to E. cloacae (Ren et al. 2010; Stover et al. 2000). After a $24 \mathrm{~h}$ incubation period of the bacterial combination, some VOCs were observed to be elevated in co-culture. Methyl 2-methylbutyrate appears to be produced by $P$. aeruginosa and has been previously reported (Filipiak et al. 2012) but is elevated in co-culture. Similarly, 2-methylbutyl acetate appears to be produced by E. cloacae and increased levels was observed in co-culture. Overexpression of indole by $E$. coli when in co-culture with $P$. aeruginosa has been observed and shown to be a virulence factor which suppresses growth of $P$. aeruginosa (Chu et al. 2012; Culotti and Packman 2014). Thus it could be the case that these metabolites are overexpressed in an attempt to induce suppression and establish dominance, although at this stage there is no direct evidence of this. Both compounds have been reported to exhibit antimicrobial activity in a study investigating the antimicrobial properties of Roman chamomile oil (Bail et al. 2009). 2-methylbutyl acetate displays antimicrobial activity towards a variety of microbes 


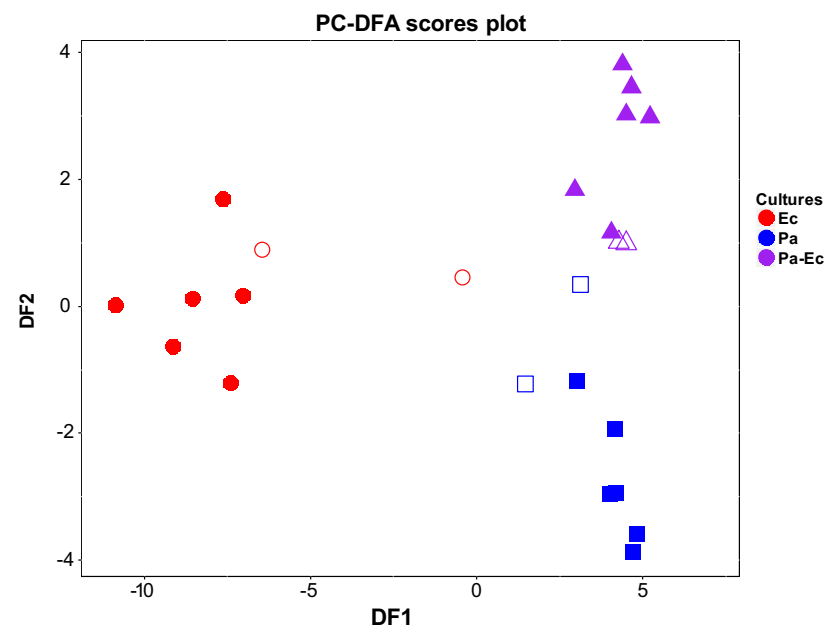

Fig. 5 PC-DFA scores validation biplot. For DFA this used 8 PCs (accounting for $64.5 \%$ of total explained variance) along with the information on the three classes used as the a priori information. Training samples from the distinct bacterial cultures are indicated as filled shapes while the projected test samples are hollow. Ec E. cloacae (circle), $P a$ P. aeruginosa (square), Pa-Ec P. aeruginosa-E. cloacae (triangle)

including $P$. aeruginosa, and thus its upregulation by $E$. cloacae may be a strategy to deter the growth of the former and may have also contributed to the delayed lag phase observed from the viable cell counts. As suppression of $P$. aeruginosa was not achieved, this may have provided the opportunity for this bacterium to reciprocate by emitting its own antimicrobial compound methyl 2-methylbutyrate. This metabolite is reported to be ineffective against $E$. coli and $K$. pneumoniae (Bail et al. 2009) and therefore may not be effective against $E$. cloacae since they belong to the same family. It has also been previously reported that the metabolism of a co-cultured species changes under detection of emitted VOCs by another species (Dow 2017). It would be interesting to investigate beyond $24 \mathrm{~h}$ to understand and gain insight into this relationship.

PC-DFA was used to visualise the relationship between the mono-cultures along with the co-culture of both bacteria. PC-DF1 which accounts for the largest group separation separated E. cloacae from $P$. aeruginosa and the second PC-DF allowed some separation of the co-culture from $P$. aeruginosa, although it was clear that the VOCs of the coculture was more similar to $P$. aeruginosa than it was to E. cloacae. VOCs found in mono- (2-methyl-1propanol, 3-methyl-1-butanol, and 1-undecene) and co-cultures (isoamyl butyrate and 2-methylbutyl acetate) were observed to be the main contributors to the separation observed along DF1 and DF2 axis of the PC-DFA scores plot.

These metabolites can be sought after in breath specimens obtained from patients and have their biomarker credentials assessed. Another potential application is to screen these compounds from results of headspace analysis of lower respiratory tract specimens obtained from patients. Although still an invasive procedure, it would eliminate the delay associated with pathogen identification and aid targeted antibiotic therapy.

\section{Conclusion}

VOCs in exhaled breath have potential for clinical translation into the clinic as proven in the case of exhaled nitric oxide used in the diagnosis of asthma. To extend this application for infection diagnosis, microbial VOCs originating from E. cloacae and P. aeruginosa mono- and co-cultures were investigated in vitro. We observed VOCs such as 2-methyl-1-propanol, 2-phenylethanol, 1-undecene and pyrrole in axenic cultures that can be utilised for bacterial species differentiation. In addition, VOCs including 2-methylbutyl acetate and methyl 2-methylbutyrate can be useful in discriminating mono- and co-cultures which may potentially translate to the distinction between mono- and polymicrobial infections. Validation of these in vitro markers in breath specimens or airway samples can potentially prove to be a clinically useful tool for elucidating causal pathogen identification and infection type discernment which may translate into appropriate antibiotic therapy regimen and improve patient outcomes.

Acknowledgements This work was funded by the European Union FP7 Marie Curie Actions, under the Industry-Academia Partnerships and Pathways (IAPP) programme (MC-IAPP BreathDx 611951). A list of all members of the BreathDx Consortium: Waqar M. Ahmed, Antonio Artigas, J. Bannard-Smith, Lieuwe D. J. Bos, Marta Camprubi, Luis Coelho, Paul Dark, Alan Davie, Emili Diaz, Gemma Goma, Timothy Felton, Stephen J. Fowler, Royston Goodacre, Hugo Knobel, Oluwasola Lawal, Jan-Hendrik Leopold, Tamara M.E. Nijsen, Pouline M. P. van Oort, Pedro Povoa, Craig Portsmouth, Nicholas J. W. Rattray, Guus Rijnders, Marcus J. Schultz, Ruud Steenwelle, Peter J. Sterk, Jordi Valles, Fred Verhoeckx, Anton Vink, Hans Weda, Iain R. White, Tineke Winters, Tetyana Zakharkina.

Data availability The data in this study are deposited in MetaboLights (https://www.ebi.ac.uk/metabolights/) study identifier MTBLS617.

\section{Compliance with ethical standards}

Conflict of interest The authors declare that they have no conflicts of interest.

Research involving with human and animal participants This article does not contain any studies with human participants or animals performed by any of the authors.

Open Access This article is distributed under the terms of the Creative Commons Attribution 4.0 International License (http://creat ivecommons.org/licenses/by/4.0/), which permits unrestricted use, 
distribution, and reproduction in any medium, provided you give appropriate credit to the original author(s) and the source, provide a link to the Creative Commons license, and indicate if changes were made.

\section{References}

Ahmed, W. M., lawal, O., Nijsen, T. M., Goodacre, R., \& Fowler, S. J. (2017). Exhaled volatile organic compounds of infection: A systematic review. ACS Infectious Diseases, 3(10), 695-710.

Allardyce, R. A., Langford, V. S., Hill, A. L., \& Murdoch, D. R. (2006). Detection of volatile metabolites produced by bacterial growth in blood culture media by selected ion flow tube mass spectrometry (SIFT-MS). Journal of Microbiological Methods, 65, 361-365.

Bail, S., Buchbauer, G., Jirovetz, L., Denkova, Z., Slavchev, A., Stoyanova, A., Schmidt, E., \& Geissler, M. (2009). Antimicrobial activities of roman chamomile oil from France and its main compounds. Journal of Essential Oil Research, 21, 283-286.

Boots, A. W., Smolinska, A., van Berkel, J. J., Fijten, R. R., Stobberingh, E. E., Boumans, M. L., Moonen, E. J., Wouters, E. F., Dallinga, J. W., \& van Schooten, F. J. (2014). Identification of microorganisms based on headspace analysis of volatile organic compounds by gas chromatography-mass spectrometry. Journal of Breath Research, 8, 027106.

Chu, W., Zere, T. R., Weber, M. M., Wood, T. K., Whiteley, M., Hidalgo-Romano, B., Valenzuela, E., JR. \& Mclean, R. J. (2012). Indole production promotes Escherichia Coli mixed-culture growth with Pseudomonas aeruginosa by inhibiting quorum signaling. Applied and Environment Microbiology, 78, 411-419.

Combes, A., Figliolini, C., Trouillet, J. L., Kassis, N., Wolff, M., Gibert, C., \& Chastre, J. (2002). Incidence and outcome of polymicrobial ventilator-associated pneumonia. Chest, 121, 1618-1623.

Culotti, A., \& Packman, A. I. 2014. Pseudomonas aeruginosa Promotes Escherichia Coli Biofilm Formation in Nutrient-Limited Medium. PLoS ONE, 9(9), e107186.

Davin-Regli, A., \& Pages, J. M. 2015. Enterobacter aerogenes and Enterobacter cloacae; versatile bacterial pathogens confronting antibiotic treatment. Frontiers in Microbiology, 6, 392.

Diraviam Dinesh, S. (2010). Artificial sputum medium. Protocol Exchange. https://doi.org/10.1038/protex.2010.212.

Dow, J. M. (2017). Diffusible signal factor-dependent quorum sensing in pathogenic bacteria and its exploitation for disease control. Journal of Applied Microbiology, 122, 2-11.

Ferrer, M., Difrancesco, L. F., Liapikou, A., Rinaudo, M., Carbonara, M., Li Bassi, G., Gabarrus, A., \& Torres, A. (2015). Polymicrobial intensive care unit-acquired pneumonia: Prevalence, microbiology and outcome. Critical Care, 19, 450.

Filipiak, W., Sponring, A., Baur, M. M., Filipiak, A., Ager, C., Wiesenhofer, H., Nagl, M., Troppmair, J., \& Amann, A. (2012). Molecular analysis of volatile metabolites released specifically by Staphylococcus aureus and Pseudomonas aeruginosa. BMC Microbiology, 12, 113.

Gehm, H. W., \& Heukelekian, H. (1935). Eosin methylene blue agar for rapid direct count of E. coli. American Journal of Public Health and the Nations Health, 25, 920-923.

Goodacre, R., Timmins, E. M., Burton, R., Kaderbhai, N., Woodward, A. M., Kell, D. B., \& Rooney, P. J. (1998). Rapid identification of urinary tract infection bacteria using hyperspectral whole-organism fingerprinting and artificial neural networks. Microbiology, 144(Pt 5), 1157-1170.

John, J. F. Jr, Sharbaugh, R. J., \& Bannister, E. R. (1982). Enterobacter cloacae: Bacteremia, epidemiology, and antibiotic resistance. Reviews of Infectious Diseases, 4, 13-28.
Jombart, T. (2008). Adegenet: A R package for the multivariate analysis of genetic markers. Bioinformatics, 24, 1403-1405.

Jombart, T., Devillard, S., \& Balloux, F. (2010). Discriminant analysis of principal components: A new method for the analysis of genetically structured populations. BMC Genetics, 11, 94 .

Junger, M., Vautz, W., Kuhns, M., Hofmann, L., Ulbricht, S., Baumbach, J. I., Quintel, M., \& Perl, T. (2012). Ion mobility spectrometry for microbial volatile organic compounds: A new identification tool for human pathogenic bacteria. Applied Microbiology and Biotechnology, 93, 2603-2614.

Kalanuria, A. A., Ziai, W., \& Mirski, M. (2014). Ventilator-associated pneumonia in the ICU. Critical Care, 18, 208.

Keller, R., Pedroso, M. Z., Ritchmann, R., \& Silva, R. M. (1998). Occurrence of virulence-associated properties in Enterobacter cloacae. Infection and Immunity, 66, 645-649.

Lawal, O., Ahmed, W. M., Nijsen, T. M. E., Goodacre, R., \& Fowler, S. J. (2017a). Exhaled breath analysis: A review of 'breath-taking' methods for off-line analysis. Metabolomics, 13, 110.

Lawal, O., Muhamadali, H., Ahmed, W., White, I. R., Nijsen, T. M. E., Goodacre, R., \& Fowler, S. J. (2017b). Headspace volatile organic compounds from bacteria implicated in ventilator-associated pneumonia analysed by TD-GC/MS. Journal of Breath Research, 12(2), 026002.

Leininger, D. J., Roberson, J. R., \& Elvinger, F. (2001). Use of eosin methylene blue agar to differentiate Escherichia Coli from other gram-negative mastitis pathogens. Journal of Veterinary Diagnostic Investigation, 13, 273-275.

Littman, D. R., \& Pamer, E. G. (2011). Role of the commensal microbiota in normal and pathogenic host immune responses. Cell Host \& Microbe, 10, 311-323.

Lourenco, C., \& Turner, C. (2014). Breath analysis in disease diagnosis: Methodological considerations and applications. Metabolites, 4, 465-498.

Martin, R., Miquel, S., Ulmer, J., Kechaou, N., Langella, P., \& Bermudez-Humaran, L. G. (2013). Role of commensal and probiotic bacteria in human health: A focus on inflammatory bowel disease. Microbial Cell Factories, 12, 71.

Mietto, C., Pinciroli, R., Patel, N., \& Berra, L. (2013). Ventilator associated pneumonia: Evolving definitions and preventive strategies. Respiratory Care, 58, 990-1007.

Neerincx, A. H., Geurts, B. P., Habets, M. F., Booij, J. A., Van Loon, J., Jansen, J. J., Buydens, L. M., Van Ingen, J., Mouton, J. W., Harren, F. J., Wevers, R. A., Merkus, P. J., Cristescu, S. M., \& Kluijtmans, L. A. (2016). Identification of Pseudomonas aeruginosa and Aspergillus fumigatus mono- and co-cultures based on volatile biomarker combinations. Journal of Breath Research, 10, 016002.

Parisi, J. T., \& Marsik, F. J. (1969). Atypical reactions of Escherichia Coli on eosin methylene blue Agar. Applied Microbiology, 18, 948.

Park, D. R. (2005). The microbiology of ventilator-associated pneumonia. Respiratory Care, 50, 742-763. (discussion 763-765).

Phillips, M. (1992). Breath tests in medicine. Scientific American, 267, 74-79.

Ren, Y., Ren, Y., Zhou, Z., Guo, X., Li, Y., Feng, L., \& Wang, L. (2010). Complete genome sequence of Enterobacter cloacae subsp. cloacae type strain ATCC 13047. Journal of Bacteriology, 192, 2463-2464.

Shestivska, V., Nemec, A., Drevinek, P., Sovova, K., Dryahina, K., \& Spanel, P. (2011). Quantification of methyl thiocyanate in the headspace of Pseudomonas aeruginosa cultures and in the breath of cystic fibrosis patients by selected ion flow tube mass spectrometry. Rapid Communications in Mass Spectrometry, 25, 2459-2467.

Smith, C. A., Want, E. J., O’maille, G., Abagyan, R., \& Siuzdak, G. (2006). XCMS: Processing mass spectrometry data for metabolite 
profiling using nonlinear peak alignment, matching, and identification. Analytical Chemistry, 78, 779-787.

Stover, C. K., Pham, X. Q., Erwin, A. L., Mizoguchi, S. D., Warrener, P., Hickey, M. J., Brinkman, F. S., Hufnagle, W. O., Kowalik, D. J., Lagrou, M., Garber, R. L., Goltry, L., Tolentino, E., WestbrockWadman, S., Yuan, Y., Brody, L. L., Coulter, S. N., Folger, K. R., Kas, A., Larbig, K., Lim, R., Smith, K., Spencer, D., Wong, G. K., Wu, Z., Paulsen, I. T., Reizer, J., Saier, M. H., Hancock, R. E., Lory, S. \& Olson, M. V. (2000). Complete genome sequence of Pseudomonas aeruginosa PAO1, an opportunistic pathogen. Nature, 406, 959-964.

Sumner, L. W., Amberg, A., Barrett, D., Beale, M. H., Beger, R., Daykin, C. A., Fan, T. W., Fiehn, O., Goodacre, R., Griffin, J. L., Hankemeier, T., Hardy, N., Harnly, J., Higashi, R., Kopka, J., Lane, A. N., Lindon, J. C., Marriott, P., Nicholls, A. W., Reily, M. D., Thaden, J. J. \& Viant, M. R. (2007). Proposed minimum reporting standards for chemical analysis: Chemical Analysis Working Group (CAWG) Metabolomics Standards Initiative (MSI). Metabolomics, 3, 211-221.
Tait, E., Perry, J. D., Stanforth, S. P., \& Dean, J. R. (2014). Identification of volatile organic compounds produced by bacteria using HS-SPME-GC-MS. Journal of Chromatographic Science, 52, 363-373.

Ubeda, C., Djukovic, A., \& Isaac, S. (2017). Roles of the intestinal microbiota in pathogen protection. Clinical and Translational Immunology, 6, e128.

Zhu, J., Bean, H. D., Kuo, Y. M., \& Hill, J. E. (2010). Fast detection of volatile organic compounds from bacterial cultures by secondary electrospray ionization-mass spectrometry. Journal of Clinical Microbiology, 48, 4426-4431.

Zscheppank, C., Wiegand, H. L., Lenzen, C., Wingender, J., \& Telgheder, U. (2014). Investigation of volatile metabolites during growth of Escherichia Coli and Pseudomonas aeruginosa by needle trap-GC-MS. Analytical and Bioanalytical Chemistry, 406, 6617-6628. 